\title{
Use of daratumumab in patients with amyloidosis
}

\author{
Participants: \\ Adriano Anzai ${ }^{1}$ \\ (iD) Armelin Utino ${ }^{1}$ \\ (D) Haroldo Katayama ${ }^{1}$ \\ (iD) Ighor A. Z. Spir ${ }^{1}$ \\ Marcio A. Lemos ${ }^{1}$ \\ (iD) Mauricio Anhesini ${ }^{1}$ \\ Oswaldo S. Tiezzi ${ }^{1}$ \\ (iD) Patricia R. N. Spir ${ }^{1}$ \\ (iD) Pericles Otani ${ }^{1}$ \\ Wanderley M. Bernardo ${ }^{2}$
}

1. Grupo de M.B. E - Presidente Prudente, Presidente Prudente, SP, Brasil 2. Coordenador do Programa Diretrizes da Associacao Medica Brasileira, Sao Paulo, SP, Brasil

Created on: September 2020

Contact:wmbernardo@usp.br

The Guidelines Project, an initiative of the Brazilian Medical Association, aims to combine information from the medical field in order to standardize producers to assist the reasoning and decision-making of doctors.

The information provided through this project must be assessed and criticized by the physician responsible for the conduct that will be adopted, depending on the conditions and the clinical status of each patient.

\section{INTRODUCTION}

The use of Daratumumab for treating amyloidosis is currently considered 'off label'.

Amyloidosis (AL), of light chain (LC) immunoglobulin, is a protein deposition disease characterized by different degrees of organic dysfunction, morbidity, and early death due to LC toxicity, usually produced by a population of clonal plasma cells. Cardiac involvement is common, and patients with severe heart failure have a poor prognosis, with a mean survival of 6 months and $40 \%$ in 1 year. Successful LC suppressive therapy, measured by a criterion of hematogenic response, is associated with better rates of organic response and survival. Although initial LC suppressive therapy provides high rates of overall hematologic response, most patients do not reach a complete hematological response to the treatment, allowing for the evolution of toxic amyloid deposition. Improvements in the organic functions involved are even less common. In addition, the underlying dyscrasia of plasma cells is not curable, and almost all patients eventually relapse, requiring additional LC suppressive therapy.

Treatment regimens for AL include proteasome inhibitors, alkylating agents, and steroids, as well as autologous stem cell transplant (HSCT) autologous in young patients.

Daratumumab is a G1k (IgG1k) monoclonal antibody of human immunoglobulin that targets the surface antigen CD38 in plasma cells and has demonstrated efficacy in multiple myeloma. Although the biology of clonal plasma cells in AL is distinct from that in multiple myeloma (lower cell proliferation), 
they express CD38, providing then a proposal for an extrapolated use of Daratumumab in Amyloidosis.

\section{CLINICAL QUESTION}

Does the use of Daratumumab in patients with Amyloidosis bring benefits in comparison to the conventional treatment?

PICO (P: population, I: intervention; C: comparison; O: outcome)

P: Patients with Amyloidosis

I: Daratumumab

C: conventional treatment

O: clinical improvement

\section{ELIGIBILITY CRITERIA}

PICO components

No restrictions to study design

No time or language restrictions

Full texts available

\section{DATABASES SEARCHED}

Medline (via Pubmed) and Cochrane Central

\section{SEARCH STRATEGY}

(amyloidosis AND daratumumab)

\section{RESULTS}

Were retrieved 59 works by combining the terms Amyloidosis and Daratumumab. Of these, we selected 8 studies that met the eligibility criteria. There is no randomized clinical trial completed up until now

\section{SELECTED EVIDENCE ${ }^{1-8}$ Gran C 20171}

Report of 2 cases of severe AL, one with severe heart failure and another with heart and kidney failure, undergoing treatment with Daratumumab. Both patients showed a rapid decrease in free light chains (FLC) in response to infusions of Daratumumab, with few associated adverse events. Very high risk of bias.

\section{Kaufman GP $2017^{2}$}

A retrospective analysis of patients with $\mathrm{AL}$ (biopsy and immunohistochemistry or mass spectroscopy) treated with intravenous Daratumumab $(16 \mathrm{mg} / \mathrm{kg} /$ week $)$ for 8 weeks, followed by 8 doses in alternate weeks, and then every 4 weeks. The doses were administered for 8 hours with $500 \mathrm{ml}$ of intravenous fluids, with the exception of the first dose, which was divided in half and administered in 2 consecutive days. If well tolerated, the doses could be administered for a minimum of 4 hours. All patients received premedication with acetaminophen $(650$ $\mathrm{mg}$ ), diphenhydramine (50 mg), and dexamethasone (20 mg). After the initial infusion, dexamethasone was reduced per medical criterion. Twenty-five patients received a median of 12 (3-35) infusions of Daratumumab. All patients were evaluated for toxicity and 24 were evaluated for hematological response. The overall rate of hematological response was $76 \%$, including complete response (CR) in 36\% and partial response in $24 \%$. The median response time was 1 month. Infusion reactions of grade 1-2 occurred in 15 patients, but no reactions of grade 3 or 4 were observed. Very high risk of bias.

\section{Sher T2016 3}

Report of 2 cases of AL, one with liver and heart failure and another with kidney failure, undergoing treatment with Daratumumab. Both patients showed a rapid decrease in free light chains (FLC) with adverse events that did not lead to the loss of adhesion. Very high risk of bias.

\section{Despande $\mathrm{S} 2020^{4}$}

A case report of a patient with involvement of multiple organs associated with nerve impairment; due to the nerve involvement, Daratumumab was chosen as the first drug, receiving the standard dose of $16 \mathrm{mg} / \mathrm{k}$ in 8 weekly infusions for 8 weeks ( 8 cycles) in association with dexamethasone $20 \mathrm{mg}$ before the Daratumumab infusion. There was no infection, reduction in serum M-peak and serum lambda free light chain, improvement of neuropathy, with a decrease of gabapentin, and improvement of liver and kidney functions

\section{Chung A $2019^{5}$}

A cohort study of 72 patients with a mean age of 67 years, previously treated for AL amyloidosis, included in the study between January 2016 and January 2019.

The patients received Daratumumab at a dose of $16 \mathrm{mg} / \mathrm{kg} /$ week for 8 weeks, and 8 additional doses every two weeks and then every 4 weeks, with 
dexamethasone (20 mg), and were evaluated retrospectively. The mean follow-up time was 27 months.

A total of 40 of the 52 evaluated patients achieved hematological response (77\%) in an average time of one month; 57 patients had cardiac involvement, and 55\% of them had a cardiac response in an average time of 3.2 months; 47 patients had renal involvement, and $52 \%$ of those evaluated had a renal response in an average time of 6 months.

The rate of overall survival in 2 years was $86 \%$.

\section{Lecumberri R $2020^{6}$}

A retrospective multicenter study on the treatment of relapsed or resistant evaluated 38 patients, $76 \%$ with cardiac involvement, $74 \%$ with renal involvement, and $42 \%$ with the involvement of $\geq 3$ organs. The average number of previous therapies was 02 . Hematological response in $72 \%$, including $28 \%$ of complete response. The cardiac response was $37 \%$ e kidney $59 \%$. The overall progression-free survival was $59 \%$ for the heart and $52 \%$ for the kidney.

\section{Schwotzer R $2019^{7}$}

A multicenter observational study of 10 patients, included in the study between August 2017 and October 2018, mean age 62.3 years, with LC Amyloidosis previously treated (resistant or relapsed) and a load of plasma cells from the bone marrow equal to or above $10 \%$, who feature as the patients with worse outcomes when compared to those below 10\%; all patients had cardiac involvement.

Daratumumab was administered at a dose of 16 $\mathrm{mg} / \mathrm{kg} /$ week for 8 weeks, followed by 8 additional doses every two weeks and a maintenance dose every 4 weeks, plus 20 to $40 \mathrm{mg}$ of dexamethasone.

The overall hematological response was $90 \%$, with $70 \%$ of high quality, reaching this response after 73 days, on average; $50 \%$ of the patients presented cardiac response in 3.8 months, on average.

After an average follow-up time of 10 months, 8 patients were still receiving medication.

\section{Sanchorawala V $2020^{8}$}

A prospective phase 2 trial of monotherapy with Daratumumab was designed to determine its safety, tolerability, and clinical and hematological responses.
22 patients had already been formerly treated for amyloidosis. There were no relevant adverse reactions during the infusion. The adverse reactions observed were respiratory infections (4 patients) and atrial fibrillation (4 patients). There was a complete hematological response and partial very good response in $86 \%$. Renal response occurred in 10 of 15 patients with renal involvement; 7 of 14 patients with cardiac involvement obtained a response. The average follow-up of the surviving patients was 20 months.

\section{QUALITY OF EVIDENCE ${ }^{9}$}

The evidence selected has a very high risk of bias (3 historical cohort studies, 2 case series, and 3 case reports), which in itself determines a very low strength. Thus, the level of scientific uncertainty is too high to ensure that the effects are produced by the intervention being tested; thus, future consistent evidence is necessary, which may even demonstrate damages and not benefits from the intervention.

Another 3 works were selected for resistant treatment/recurrence of amyloidosis using Daratumumab, two of them retrospective and one in phase 2 of the study, with very low strength of evidence.

\section{SYNTHESIS OF EVIDENCE}

The use of Daratumumab in patients with clinical symptoms of Amyloidosis currently should not be recommended due to the weakness of the evidence available to support it.

In patients with resistant or recurrent Amyloidosis, observational studies have demonstrated good results with the use of Daratumumab associated with Dexamethasone.

\section{PERSPECTIVES}

Currently, there are 8 clinical trials registered on ClinicalTrials.gov studying the use of Daratamumab for the treatment of patients with Amyloidosis ${ }^{10}$. Among these, there is a randomized clinical trial with 416 participants comparing the drug with the standard treatment, which should be completed in $2024^{11}$. 


\section{REFERENCES}

1. Gran C, Gahrton G, Alici E, Nahi H. Case Report: Treatment of light-chain amyloidosis with daratumumab monotherapy in two patients. Eur | Haematol 2017. doi: 10.1111/ejh.13008. [Epub ahead of print]. PMID: 29226427.

2. Kaufman GP, Schrier SL, Lafayette RA, Arai S, Witteles RM, Liedtke M. Daratumumab yields rapid and deep hematologic responses in patients with heavily pretreated AL amyloidosis. Blood 2017; 130: 900-902. doi: 10.1182/blood-2017-01-763599. Epub 2017 Jun 14. PMID: 28615223.

3. Sher $T$, Fenton $B$, Akhtar A, Gertz MA. First report of safety and efficacy of daratumumab in 2 cases of advanced immunoglobulin light chain amyloidosis. Blood 2016; 128(15):1987-1989. Epub 2016 Aug 19. PMID: 27543437.

4. Deshpande S, Gertz MA, Dispenzieri A, Kumar SS, Parikh SA, Muchtar E. Daratumumab as successful initial therapy for $A L$ amyloidosis with nerve involvement. Leuk Lymphoma. 2020;61(7):1752-1755. doi:10.1080/104281 94.2020.1737688. PMID: 32159403

5. Chung A, Kaufman GP, Sidana S, et al. Organ responses with daratumumab therapy in previously treated AL amyloidosis. Blood Adv. 2020;4(3):458-466. doi:10.1182/bloodadvances.2019000776. PMID: 32027745

6. Lecumberri R, Krsnik I, Askari E, et al. Treatment with daratumumab in patients with relapsed/refractory $A \mathrm{~L}$ amyloidosis: a multicentric retrospective study and review of the literature. Amyloid. 2020;27(3):163-167. doi:10.
1080/13506129.2020.1730791. PMID: 32106714

7. Schwotzer R, Manz MG, Pederiva S, et al. Daratumumab for relapsed or refractory $\mathrm{AL}$ amyloidosis with high plasma cell burden. Hematol Oncol. 2019;37(5):595-600. doi:10.1002/hon.2677. PMID: 31486522

8. Sanchorawala V, Sarosiek S, Schulman A, et al. Safety, tolerability, and response rates of daratumumab in relapsed $\mathrm{AL}$ amyloidosis: results of a phase 2 study. Blood. 2020;135(18):1541-1547. doi:10.1182/ blood.2019004436. PMID: 31978210

9. Guyatt G, Gutterman D, Baumann MH, Addrizzo-Harris D, Hylek EM, Phillips B et al. Grading strength of recommendations and quality of evidence in clinical guidelines: report from an american college of chest physicians task force. Chest 2006; 129: 174-81. PMID: 16424429.

10. Ensaios clínicos registrados de Amiloidose e Daratamumab no Clinical Trials.gov. Disponível em: https://clinicaltrials.gov/ct2/ results?term=daratumumab\&cond=amyloidosis

11. A Study to Evaluate the Efficacy and Safety of Daratumumab in Combination With Cyclophosphamide, Bortezomib and Dexamethasone (CyBorD) Compared to CyBorD Alone in Newly Diagnosed Systemic Amyloid Lightchain (AL) Amyloidosis. Available from: https://clinicaltrials.gov/ct2/show/ NCT03201965?term=cybord+daratumumab\&cond=amyloidosis\&rank=1 